\title{
An Algorithm to Sample an Anatomy With Uncertainty
}

\author{
Cesare Corrado ${ }^{1}$, Steven Williams ${ }^{1}$, Iain $\mathrm{Sim}^{1}$, Sam Coveney ${ }^{2}$, Mark O’Neill ${ }^{1}$, Richard Wilkinson ${ }^{2}$, \\ Jeremy Oakley ${ }^{2}$, Richard Clayton ${ }^{2}$, Steven Niederer ${ }^{1}$ \\ 1 King's College London, London, United Kingdom \\ ${ }^{2}$ University of Sheffield, Sheffield, United Kingdom
}

\begin{abstract}
Image artefact, resolution and contrast all impact our ability to quantify anatomy. In the context of patientspecific simulations this uncertainty in shape can lead to uncertainty in model predictions. We propose and apply a method for quantifying uncertainty in shape and demonstrate the impact of uncertain shape on activation time predictions in a model of cardiac electrophysiology activation.
\end{abstract}

\section{Introduction}

Patient-specific models of the heart are gaining importance in the treatment of heart diseases as they help to predict procedure outcomes and guide ablation targets, [1]. Recently, we developed and validated a novel method to generate patient-specific models of left atrial electrophysiology from clinical measurements [2] and tested its ability to differentiate aberrant activation patterns [3]. Clinical measurements, however, are affected by noise; hence, quantifying how measurement uncertainty affects model predictions represents an important step in communicating the confidence of model predictions to cardiologists. An important building block in this process is to consider the impact of uncertainties in the measurements of atrial geometry that are used to generate the mesh on which the model is solved. In this work, we propose a method to quantify the uncertainty on the anatomy of the left atrium. We then show the effect of these anatomical uncertainties on simulations of local activation times.

\section{Method}

In the next subsections, we introduce a method to characterize the uncertainty about the true anatomy $\mathbf{X}$ given an observations $\mathbf{X}_{\mathrm{obs}}$, i.e., find $p\left(\mathbf{X} \mid \mathbf{X}_{\mathrm{obs}}\right)$. Dealing with the uncertainty in the high-dimensional space where $\mathbf{X}$ is located is computationally expensive and often this complexity furnishes information about the uncertainty that is negligible. Hence, we characterise the uncertainty through its principal components, [6].

\subsection{Anatomies}

We recorded a training set of $\mathrm{M}=17$ left atrial anatomy using an electro-anatomical mapping system (EAM, St Jude Velocity) and a test set of 4 left atrial anatomies using a different EAM (Biosense Carto). On each of the 21 anatomies, we manually clipped the pulmonary veins $(\mathrm{PV})$ and the left atrial appendage (LAA). We thus obtained anatomies described by $7021 \pm 1661$ points (training set) and by $9209 \pm 2368$ (test set) in an irregular triangle shell mesh. Next, we aligned each anatomy to a reference atlas [4] formed by $N_{\mathrm{p}}=6132$ vertices. We then registered the atlas to each anatomy, by minimising the distance between the two manifolds using Deformetrica [5]. Each anatomy could then be described by the same fixed number of points.

We denote the vector $\left(\left[x_{1}, y_{1}, z_{1}, \ldots, x_{N_{\mathrm{p}}}, y_{N_{\mathrm{p}}}, z_{N_{\mathrm{p}}}\right]^{T}\right)$ of the $\mathrm{x}, \mathrm{y}$ and $\mathrm{z}$ point coordinates of the deformed atlas obtained by unrolling the point coordinates with $\mathbf{X}_{\mathrm{obs}} \in$ $\mathbb{R}^{3 \mathbf{N}_{\mathrm{p}}}$; with $\mathbf{X} \in \mathbb{R}^{\mathbf{3} \mathbf{N}_{\mathrm{p}}}$ the anatomy in the absence of uncertainty and with $\mathbf{e} \in \mathbb{R}^{3 N_{\mathrm{p}}}$ a random vector representing the uncertainty in observations of the anatomy, such that: $\mathbf{X}_{\mathrm{obs}}=\mathbf{X}+\mathbf{e}$. We further assume a normally distributed uncertainty: $\mathbf{e} \sim N\left(0, \Sigma_{\mathbf{X}}\right)$, with a mean of zero and a covariance matrix $\Sigma_{\mathbf{X}}$, with the correlation depending on the relative spatial distance.

\subsection{Principal component analysis (PCA)}

We reduced the number of parameters representing uncertainty in the anatomy by describing the anatomy as a linear combination of a reference mean value $\boldsymbol{\mu}$ and of $N_{\text {modes }} \ll 3 N_{\mathrm{p}}$ modes:

$$
\mathbf{X} \simeq \boldsymbol{\mu}+\mathbf{U} \boldsymbol{\lambda}
$$

We determined the modes that capture the largest variability on the atrial anatomies with the principal component analysis (PCA) [6] on a training set, reducing the dimensionality of the problem to $N_{\text {modes }}=16$ independent parameters. 
We first evaluated the empirical mean $\boldsymbol{\mu}$ :

$$
\boldsymbol{\mu}=\frac{1}{\mathrm{M}} \sum_{\mathrm{k}=1}^{\mathrm{M}} \mathbf{X}_{\mathrm{obs}}^{\mathrm{k}}
$$

Next, we build the matrix $A_{\mathrm{obs}} \in \mathbb{R}^{3 N_{\mathrm{p}} \times \mathrm{M}}$ where the k-th column is obtained as: $A_{\mathrm{obs} * \mathrm{k}}=\mathrm{X}_{\mathrm{obs}}^{\mathrm{k}}-\boldsymbol{\mu}$ and we evaluated the principal axes [6] with the singular value decomposition (SVD):

$$
A_{\mathrm{obs}}=U \Theta V^{T}
$$

Here $U \in \mathbb{R}^{3 N_{\mathrm{p}} \times(\mathrm{M}-1)}$ is the matrix of the left singular vectors and coincides with the principal axes, and $\Theta \in$ $\mathbb{R}^{(\mathrm{M}-1) \times(\mathrm{M}-1)}$ is the diagonal matrix of the singular values $\Theta_{\mathrm{i}}$. The variances $\sigma_{\mathrm{i}}^{2}$ captured by each mode are obtained as follows:

$$
\sigma_{\mathrm{i}}^{2}=\frac{\Theta_{\mathrm{i}}^{2}}{\mathrm{M}-1}
$$

and depicted in figure 1 .

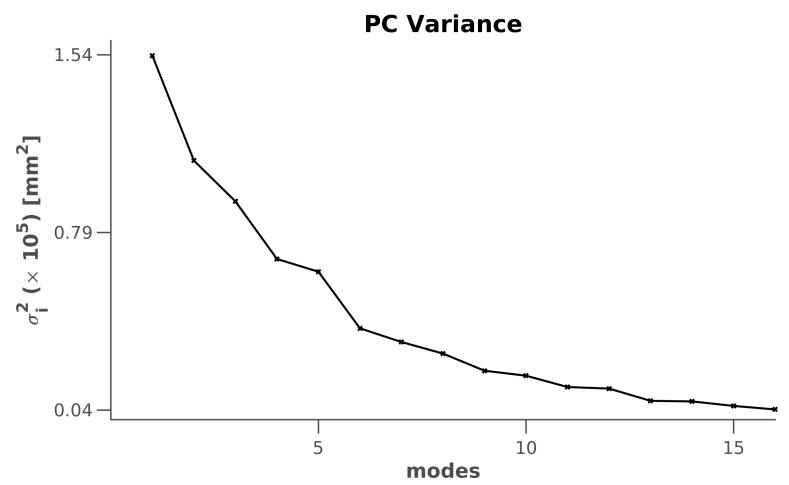

Figure 1. Plot of the variances $\sigma_{\mathrm{i}}^{2}$ captured by each mode

\subsection{Error propagation}

We decompose a new observation $\mathbf{X}_{\mathrm{obs}}^{*}$ as the sum of the reconstruction from the principal component representation, the truncation error and the uncertainty:

$$
\mathbf{X}_{\mathrm{obs}}^{*}=\mathbf{X}^{*}+\mathbf{e}=\boldsymbol{\mu}+\mathbf{U} \boldsymbol{\lambda}^{*}+\mathbf{e}_{\perp}+\mathbf{e}
$$

here the vector $\boldsymbol{\lambda}^{*} \in \mathbb{R}^{N_{\text {modes }}}$ represents the reduced representation of $\mathbf{X}^{*}$, and $\boldsymbol{e}_{\perp}$ represents the truncation error in the absence of uncertainty. Even though deterministic, the truncation error $\boldsymbol{e}_{\perp}$ is unknown and hence modelled with a probability distribution: $\boldsymbol{e}_{\perp} \sim N\left(0, \Sigma_{\boldsymbol{e}_{\perp}}\right)$. Finally, we define the global error as follows: $\boldsymbol{e}_{\mathrm{TOT}}=\mathbf{e}+\boldsymbol{e}_{\perp}$, with distribution: $\boldsymbol{e}_{\text {TOT }} \sim N\left(0, \Sigma_{\boldsymbol{e}_{\text {TOT }}}\right)$ where $\Sigma_{\boldsymbol{e}_{\text {Tот }}}=\Sigma_{\mathbf{X}}+\Sigma_{\boldsymbol{e}_{\perp}}$ and we introduce a model for $\Sigma_{\boldsymbol{e}_{\mathrm{TOT}}}$.

\subsection{Prior and posterior distribution on $\lambda^{*}$}

$\lambda^{*}$ prior distribution We assume the prior distribution $\lambda^{*} \simeq N\left(0, \Sigma_{\boldsymbol{\lambda}}\right)$ and $\Sigma_{\boldsymbol{\lambda}}=\Theta^{T} \alpha^{2} \Theta$. Using the samples from the training set, we then evaluate $\alpha^{2}$ and prove the existence of variables $\hat{\boldsymbol{\lambda}}^{i} \sim N\left(0, \alpha^{2} I\right)$, such that $\boldsymbol{\lambda}^{i}=$ $\Theta \hat{\boldsymbol{\lambda}}^{i}$. For each sample, we have:

$$
\hat{\boldsymbol{\lambda}}^{i}=\Theta^{-1} U^{T}\left(\mathbf{X}_{\mathrm{obs}}^{\mathbf{i}}-\boldsymbol{\mu}\right), i=1 \ldots M
$$

If the null hypothesis holds, $\hat{\lambda}_{\mathrm{j}}^{i}, i=1 \ldots M, j=$ $1 \ldots N_{\text {modes }}$ represent $N_{\text {dof }}=N_{\text {modes }} \times M$ realizations of $N\left(0, \alpha^{2} I\right)$. Next, we approximate $\alpha^{2}$ with the sampling covariance, $\mathrm{s}^{2}$ :

$\mathrm{s}^{2}=\frac{1}{N_{\text {dof }}-1} \sum_{i=1}^{M} \sum_{j=1}^{N_{\text {modes }}}\left(\hat{\lambda}_{\mathrm{j}}^{i}-\mu\right)^{2}, \mu=\frac{1}{N_{\text {dof }}} \sum_{i=1}^{M} \sum_{j=1}^{N_{\text {modes }}} \hat{\lambda}_{\mathrm{j}}^{i}$

Finally, we performed a Kolmogorov Smirnov test to check for normality, and found no evidence to reject the null hypothesis $(\mathrm{p}=0.56)$. The QQ plot showed excellent agreement with a standard normal distribution (not shown).

$\lambda^{*}$ posterior distribution Being the problem conjugate we obtain a normal posterior distribution $N\left(\boldsymbol{\mu}_{\boldsymbol{\lambda}, \text { post }}, \Sigma_{\boldsymbol{\lambda}, \text { post }}\right)$, and Bayes formula $p\left(\boldsymbol{\lambda}^{*} \mid \mathbf{X}_{\text {obs }}^{*}\right) \propto \mathbf{p}\left(\mathbf{X}_{\mathrm{obs}}^{*} \mid \boldsymbol{\lambda}^{*}\right) \mathbf{p}\left(\boldsymbol{\lambda}^{*}\right)$ yields:

$p\left(\boldsymbol{\lambda}^{*}\right) \sim N\left(0, \Sigma_{\boldsymbol{\lambda}}\right)$
$p\left(\mathbf{X}_{\mathrm{obs}}^{*} \mid \boldsymbol{\lambda}^{*}\right) \sim \mathbf{N}\left(\boldsymbol{\mu}+\mathbf{U} \boldsymbol{\lambda}^{*}, \boldsymbol{\Sigma}_{\mathbf{e}_{\mathrm{ToT}}}\right)$
$p\left(\boldsymbol{\lambda}^{*} \mid \mathbf{X}_{\mathrm{obs}}^{*}\right) \propto \exp \left(-\frac{\boldsymbol{\lambda}^{*{ }^{\mathbf{T}}} \boldsymbol{\Sigma}_{\boldsymbol{\lambda}, \text { post }}^{-\mathbf{1}} \boldsymbol{\lambda}^{*}-\mathbf{2} \boldsymbol{\lambda}^{*^{\mathbf{T}}} \boldsymbol{\Sigma}_{\boldsymbol{\lambda}, \text { post }}^{-\mathbf{1}} \boldsymbol{\mu}_{\boldsymbol{\lambda}, \text { post }}+\mathbf{a}}{\mathbf{2}}\right)$

and we thus obtain the posterior distribution for $\lambda^{*}$ :

$$
\begin{aligned}
& p\left(\boldsymbol{\lambda}^{*} \mid \mathbf{X}_{\mathrm{obs}}^{*}\right) \sim \mathbf{N}\left(\boldsymbol{\mu}_{\boldsymbol{\lambda}, \text { post }}, \boldsymbol{\Sigma}_{\boldsymbol{\lambda}, \text { post }}\right) \\
& \Sigma_{\boldsymbol{\lambda}, \text { post }}=\left(\Sigma_{\boldsymbol{\lambda}}^{-1}+U^{T} \Sigma_{\left.\boldsymbol{e}_{\text {TOT }}^{-1} U\right)^{-1}}\right. \\
& \boldsymbol{\mu}_{\boldsymbol{\lambda}, \text { post }}=\Sigma_{\boldsymbol{\lambda}, \text { post }} U^{T} \Sigma_{\boldsymbol{e}_{\text {TOT }}^{-1}}\left(\mathbf{X}_{\mathrm{obs}}^{*}-\boldsymbol{\mu}\right)
\end{aligned}
$$

\subsection{Sampling}

Once a new anatomy $\mathbf{X}_{\mathrm{obs}}^{*}$ is measured with EAM, we draw $N_{\text {samples }}$ i.i.d. samples from $p\left(\boldsymbol{\lambda}^{*} \mid \mathbf{X}_{\text {obs }}^{*}\right)$, obtained by applying (1); next, we obtain the anatomy samples $\left(\mathbf{X}^{\mathbf{1}}, . ., \mathbf{X}^{\mathbf{N}_{\text {samples }}}\right)$ with the formula: $\mathbf{X}^{\mathbf{k}}=\boldsymbol{\mu}+\mathbf{U} \boldsymbol{\lambda}^{\mathbf{k}}$

\section{Results}

We sample from the uncertainty distribution of each of the 4 anatomies depicted in figure 2. We assumed the fol- 

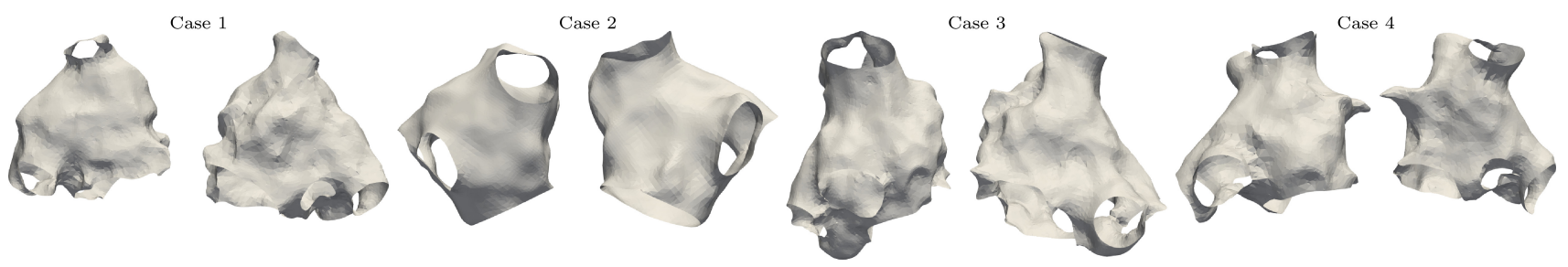

Figure 2. Anatomies

lowing form for $\Sigma_{\boldsymbol{e}_{\mathrm{TOT}}}$ :

$$
\Sigma_{\boldsymbol{e}_{\mathrm{TOT}}}^{i_{x_{\mathrm{k}},}, j_{x_{1}}}=\nu^{2} \exp \left(-\left(\frac{d_{\mathrm{ij}}}{l}\right)^{2}\right) \delta_{x_{\mathrm{k}}, x_{1}}
$$

Here $\nu^{2}$ represents the characteristic scale of the covariance, $l$ represents the characteristic length of the space correlation, $d_{\mathrm{ij}}$ represents the geodesic distance between points $\mathrm{i}$ and $\mathrm{j}, \delta_{x_{\mathrm{k}}, x_{1}}$ represent the Dirac delta between the space components $x_{\mathrm{k}}$ and $x_{1}$ and index $i_{x_{\mathrm{k}}}$ represents the entry in $\Sigma_{\boldsymbol{e}_{\mathrm{TOT}}}$ corresponding to node $\mathrm{i}$ and component $x_{\mathrm{k}}$.

As the typical discrepancy between anatomies obtained with EAM and anatomies obtained with MRI scan is $\simeq 5 \mathrm{~mm}$ [7], in this work we considered the following characteristic values: $\nu^{2}=[25,49,100,225,784] \mathrm{mm}^{2}$, while we arbitrarily chose a width $l$ equal to $10 \mathrm{~mm}$.

Next, for each sample, we computed LATs with a graph-based eikonal model [8] and the modified MitchellSchaeffer (mMS) ionic model [9]. For the model parameter values we selected $\tau_{\text {in }}=0.1 \mathrm{~ms}, \tau_{\text {out }}=2.5 \mathrm{~ms}$ and $v_{\text {gate }}=0.1$ and we chose an isotropic diffusivity of $\sigma_{\mathrm{m}}=1.0 \mathrm{~cm}^{2} / \mathrm{s}$, consistent with $[2,10]$. To take into account the effect of low mesh resolution on the propagation through a graph, we set the corrective coefficient to $\delta=0.92$. This value furnished the minimum RMS between the LATs evaluated with the eikonal model and those computed solving the monodomain equations with the finite elements method (FEM) on a discretized domain characteristic size $h \simeq 300 \mu \mathrm{m}$.

Next, we evaluated the expected value and the standard deviation of LATs with the Monte Carlo [11] formula. For each anatomy and for each value of $\nu^{2}$, we draw $N_{\text {samples }}=12,000$ i.i.d. anatomy samples; overall, the procedure required $\sim 70$ minutes for a single case and for a single value of $\nu^{2}$. The $l_{2}$ norm of the difference between the expected value evaluated with $12 \mathrm{k}$ samples and the expected values evaluated with $10 \mathrm{k}$ samples was $2.6 \mathrm{~ms} \pm 0.58 \mathrm{~ms}$, or $\sim 1 \%$ of the total activation time. Figure 3 shows the distribution of expected value for LAT, and the standard deviation for each of the 4 patients and when $\nu=28 \mathrm{~mm}$.

Figure 4 shows the maximum value of LAT standard deviation as a function of the uncertainty standard deviation $\nu$; each line represents a patient.

\section{Discussion}

In this work, we have presented a method to evaluate the statistics of a space-dependent variable and to infer a shape when the data quality is not high. We parameterised the uncertainty on the domain using PCA. When the uncertainty affects the computational domain, a widely used approach is represented by stochastic collocation techniques, $[12,13]$. This approach, however, does not take into account any spatial correlation on the domain uncertainty. Indeed, this uncertainty is described by parametrising the uncertain domain through a linear composition of shape functions, weighted with Karhunen-Loeve (KL) approximation. This is not the case with the approach we presented: first, the spatial correlation is implicitly described by principal axes; second, among all the possible orthogonal shape functions that represent space uncertainty, the principal axes are those that minimise the truncation error; third, the coefficients of the reduced representation of $\mathbf{X}$ are mutually independent and ordered: we can thus capture the largest variability (in the statistical sense) keeping the same accuracy using a smaller number of parameters.

\section{Conclusions}

We have developed a method to sample from an anatomy probability distribution and to infer model uncertainty. We took advantage of a PCA decomposition for the anatomy, obtaining a method computationally efficient.

\section{Acknowledgements}

This work was supported by the EPSRC (EP/P101268X/ 1), the Wellcome centre and the Department of Health via the National Institute for Health Research (NIHR) comprehensive Biomedical Research Centre award to Guy's \& St Thomas' NHS Foundation Trust in partnership with King's College London and King's College Hospital NHS Foundation Trust.

\section{References}

[1] Colli Franzone P, Pavarino L, Savarè G. Computational electrocardiology: mathematical and numerical modelling. Complex systems in Biomedicine Springler, 2006:187-241. 


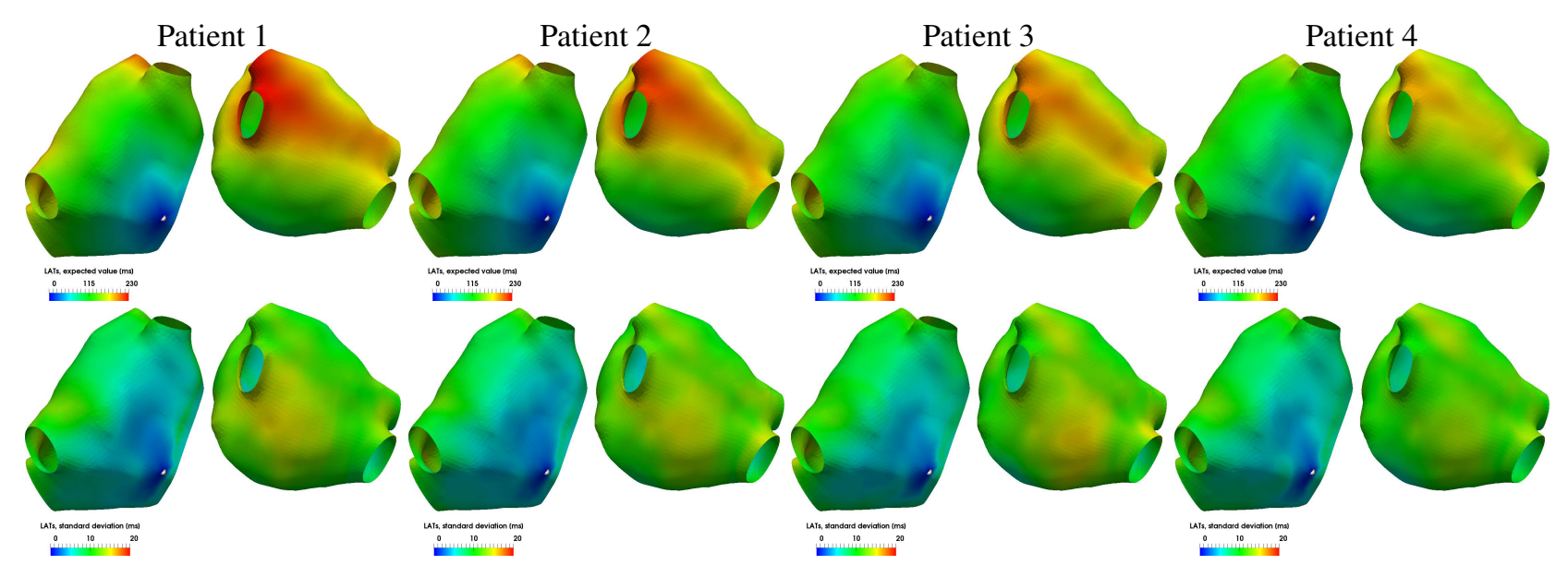

Figure 3. Top: Distribution of LATs expected values for patients 1,2,3,4. Bottom:Distribution of LATs standard deviation for patients $1,2,3,4$.

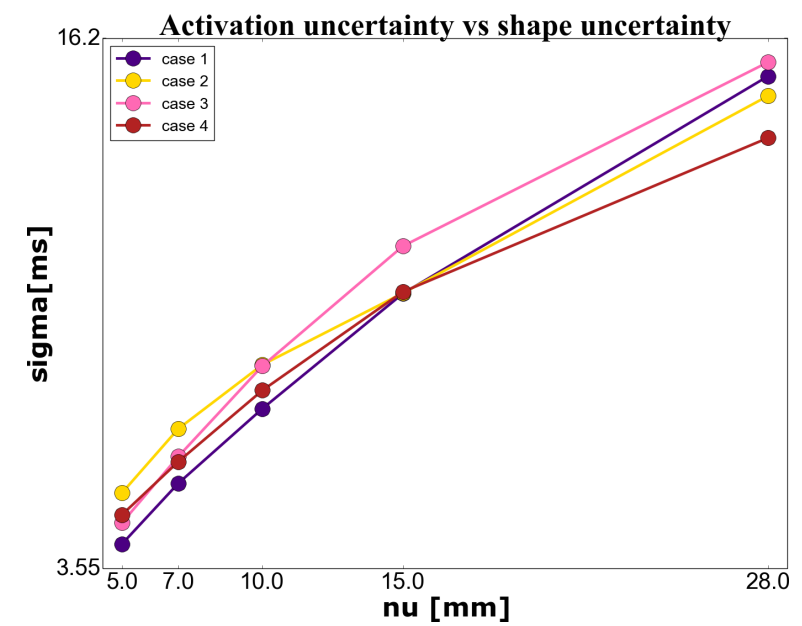

Figure 4. Maximum value of the standard deviation on LATs as a function of the standard deviation of the uncertainty $\nu$; each line represents a patient.

[2] Corrado C, et al. A work flow to build and validate patient specific left atrium electrophysiology models from catheter measurements. Medical Image Analysis 2018; 47:153-163.

[3] Corrado C, et al. A predictive personalised model for the left atrium. 2017 Computing in Cardiology (CinC) 2017.

[4] Tobon-Gomez C, et al. Left atrial segmentation challenge: a unified benchmarking framework. Statistical Atlases and Computational Models of the Heart. Imaging and Modelling Challenges: 4th International Workshop, STACOM 2013, Held in Conjunction with MICCAI 2013, Nagoya, Japan, September 26, 2013. Revised Selected Papers Springer Berlin Heidelberg, 2014; 1-13.

[5] www.deformetrica.org

[6] Hotelling H. Analysis of a complex of statistical variables with principal components. Journal of Educational Psychology 1993; 24:417-441.

[7] Nguyen, U C, et al. A novel approach for left ven- tricular lead placement in cardiac resynchronization therapy: Intraprocedural integration of coronary venous electroanatomic mapping with delayed enhancement cardiac magnetic resonance imaging. Heart Rhythm 2017; 14(1):110-119.

[8] Corrado C, Zemzemi N. A conduction velocity adapted eikonal model for electrophysiology problems with reexcitability evaluation. Medical Image Analysis 2018; 43:186-197.

[9] Corrado C, Niederer S. A two-variable model robust to pacemaker behaviour for the dynamics of the cardiac action potential. Mathematical Biosciences 2016; 281:46-54.

[10] Corrado C, et al. Predicting spiral wave stability by personalized electrophysiology models. 2016 Computing in Cardiology (CinC) 2016.

[11] Kroese, D, et al. Why the Monte Carlo method is so important today. Wiley Interdisciplinary Reviews: Computational Statistics John Wiley \& Sons, Inc, 2014:386-392.

[12] Castrillón-Candás J, Nobile F, Tempone R. Analytic regularity and collocation approximation for elliptic PDEs with random domain deformations. Computers \& Mathematics with Applications 2016; 71(6):1173-1197

[13] Sankaran, S and Marsden, AL. A stochastic collocation method for uncertainty quantification and propagation in cardiovascular simulations. ASME. J Biomech Eng 2011; 33(3):031001-031001-12

Address for correspondence:

Cesare Corrado

Dep. of Biomedical Engineering; St Thomas' Hospital, SE17EH, London (UK)

cesare.corrado@kcl.ac.uk 